\title{
Current Status of QEEG and Neurofeedback in the Treatment of Clinical Depression
}

\author{
By \\ Jonathan Walker, M.D. $^{\text {ab }}$ \\ a \\ Robert Lawson, M.S. \\ Gerald Kozlowski, Ph.D. ${ }^{\text {ab }}$ \\ ${ }^{a}$ Neurotherapy Center of Dallas \\ and \\ ${ }^{\mathrm{b}}$ University of Texas Southwestern Medical School, Dallas \\ Email: admin@neurotherapydallas.com \\ Contemporary Topics in Neurofeedback \\ Edited by \\ James R. Evans, PhD
}




\section{Introduction}

Depression is an almost universal experience. Fortunately, it usually remits spontaneously after a period of time. Unfortunately, it does not spontaneously remit in many individuals, and they are diagnosed as being "clinically" depressed, requiring treatment to be able to have an acceptable quality of life and be able to work. Several depression subtypes have been defined based on their semiology (Table I). We have indicated the most common abnormalities found in our clinic for each type of depression, and indicated the type of neurofeedback training that most effectively remediates the depression and prevents further episodes, in our experience. Note that alpha asymmetry appears in most cases to be a state marker rather than a trait marker, and is not seen with the linked ears reference used in all commercially available databases. One must use a CZ reference to identify the asymmetry and $\mathrm{Cz}$ is not an inactive reference. However, alpha asymmetry may represent a trait marker in some patients, and alpha asymmetry training may produce long-lasting remissions in some patients.(Baehr \& Baehr, 1997; Baehr, Rosenfeld \& Baehr, 1997; Rosenfeld, Baehr E., Baehr, R., Gottlieb, \& Rangarndth, 1996; Earnest, 1999; Rosenfeld, 1999; Baehr, Rosenfeld, Baehr \& Earnest, 1999)

\section{The Neurobiology of Depression}


Adolphs and Tranel (2004) reviewed the neurobiology of emotions generally and in depression and mania, specifically. Neural structures that process emotions in humans include the left and right hemispheres, amygdala, orbitofrontal cortex, basal ganglia, cingulated gyrus and hippocampus. The left cerebral hemisphere is more involved in positive emotions, and the right hemisphere is more involved in negative emotions. Davidson and Irwin (1999) posited an approach/withdrawal dimension, correlating increased right hemisphere activation with increases in withdrawal behavior (including emotions such as fear or sadness, as well as depressive tendencies), and increased left hemisphere activity with increase in approach behaviors (including emotions such as happiness). An important key issue for neurofeedback therapists is what exactly constitutes "activation." We will address this in the QEEG section of this chapter. Major depression has been associated with damage to the frontal lobes, especially the left frontal pole (Starkstein \& Robinson, 1991). PET studies have shown that a region under the genu of the corpus callosum, the subcallosal gyrus, is consistently underactivated in patients with depression (Ongur, Drevets, \& Price, 1998). As reviewed by Liotti and Mayberg (2001) depression also is associated with hypometabolism in the cingulate cortex and occasionally in other areas such as the orbitofrontal, insular, and anterior temporal cortices, amygdalae, basal ganglia, and thalamus. 
Reports on increased activation of any particular area have not consistently been associated with depression. Liotti and Mayberg (2001) found that induced sadness was associated with metabolic activation of limbic and paralimbic regions, ventral anterior cingulate, insula, and cerebellar vermis. Furthermore. Mayberg, Brannon, Tekell, Silva \& McGinnis (2000)_found that recovery from depression was associated with activation in dorsal cortical, inferior parietal, dorsal anterior cingulate, and posterior cingulate areas. There were concomitant decreases in ventral limbic and paralimbic areas, including the subgenual cingulate and ventral, mid- and posterior insula, hippocampus, and hypothalamus.

Anxiety, on the other hand, correlates with increased regional cerebral blood flow $(\mathrm{rCBF})$ in posterior cingulate and bilateral inferior parietal lobules. Since comorbid depression and anxiety are common, it is important to recognize the different areas that are activated or inhibited by both depression and anxiety. These relationships are depicted in Table I, illustrating the presumed role of these areas in producing or inhibiting depression and anxiety or their opposites, happiness/calmness. While this model is admittedly over simplified and all details not proven, it does serve a heuristic purpose for approaching depression and anxiety using neurofeedback. This approach will be discussed in the neurofeedback section.

\section{QEEG and Depression}


EEG and QEEG are excellent approaches to measuring activation of cerebral cortical areas, but do not access all cortical areas (when the 10/20 system is used). Available databases do not reliably indicate activation or inhibition of subcortical structures, unless LORETA is used. There are few studies of depression using LORETA, and a coherent description of subcortical structures activated or inhibited in depression does not exist.

What exactly constitutes an activated EEG for a given brain area? Most people would agree that delta and theta rhythms indicate a hypoactive state. Alpha rhythms are associated with more activation, and increasing levels of beta are generally associated with even activation. Table II indicates the clinical states typically associated with frontal rhythms as seen in the clinical setting. A QEEG is necessary to determine whether the rhythms seen are normal, low, or high compared to the normal population. Generally in neurofeedback, the goal is to normalize the activity by uptraining low values, downtraining high values, and leaving normal values alone.

Commercially available databases usually use a linked ears reference, as it approximates a neutral reference in most cases. As mentioned before, it is important to recognize that $\mathrm{CZ}$ is not a neutral reference, i.e. it is an active reference. For this reason, no available databases use a $\mathrm{CZ}$ reference, 
A second technical point is also important with reference to using neurofeedback to treat depression (or other disorders) and to measure EEG changes resulting from treatment. Push-pull amplifiers measure the difference in potential between two electrodes. If one of the electrodes is non-active (a reference electrode), then one can measure the potential at the active electrode. If both references are active, it is not possible to know which electrode has the higher or lower value, or whether both electrodes have a higher or lower potential than a true reference electrode. Monopolar training, utilizes a non-active (reference) electrode; therefore, it gives one a certain measure of the effectiveness of training at the active electrode. Bipolar training, where both references are active, does not give an accurate measure of what is happening at either electrode. A change in potential difference caused by bipolar training could represent 1 of 6 possibilities:

A

1) No change at electrode $A$, increase at electrode $B$

2) No change at electrode $B$, increase at electrode $A$

3) Increase at both electrodes, but more at B than $A$

4) Increase at both electrodes, but more at A than B

5) Decrease at both electrodes, but more at $B$ than $A$

6) Decrease at both electrodes, but more at A than B

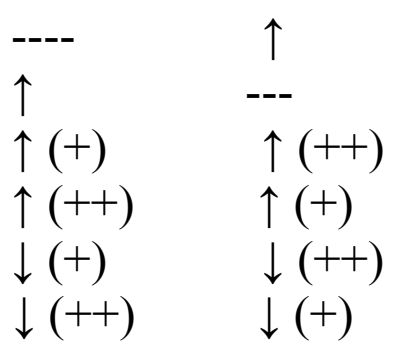

Similarly, a decrease in potential caused by bipolar training could represent 1 of 6 possibilities. It is even more difficult to measure the result of asymmetry training, since it involves a ratio of increases or decreases. 
A third technical point has to do with the choice of what to uptrain or downtrain. Since the neurobiological studies of depression indicate that left frontal activation is important in being happy rather than depressed, it would seem that training beta2 $(15-18 \mathrm{~Hz})$ activity would be a more direct way to train the relevant area than would training to normalize symmetry between right and left frontal alpha, which might or might not be associated with an increase in left frontal activation in the $15-18 \mathrm{~Hz}$ range. This might account for failures in alpha asymmetry training for long-term prevention of depression in some cases.

E. R. John and his colleagues (Prichep, Lieber, \& John, 1986) were the first to describe QEEG abnormalities associated with depression and depression subtypes. No one variable could identify a depressive disorder, but using 23 variables, in a multivariate analysis, they were able to correctly identify depressed patients with an $84 \%$ accuracy. The largest variances from normal included absolute power in the frontal-temporal regions (especially on the left), power symmetry in the temporal and fronto-temporal regions, and the combined features for coherence in the anterior regions. Bipolar patients could be discriminated from unipolar patients using 7 input variables. The variables that accounted for the most variance were left parietal-occipital beta power (deficient in unipolar, excess in bipolar); left hemisphere alpha power (deficient in bipolar), and anterior coherence (decreased in theta band in unipolar and decreased in beta band in bipolar 
patients). Accuracy was $87 \%$ for identifying unipolar individuals and $90 \%$ for identifying bipolar individuals. Lieber and Newbury (1988) delineated the subtypes of unipolar depression.

Lawson, Barnes, Bodenhamer-Davis, \& Reed, (2000) have developed a new asymmetry metric that emphasizes coherence asymmetry rather than power asymmetry. Alpha coherence asymmetry is calculated as:

(F3/Cz alpha coherence --F4/Cz alpha coherence) (F3/Cz alpha coherence $+\mathrm{F} 4 / \mathrm{Cz}$ alpha coherence)

Therefore, alpha coherence asymmetry shows the differences between the amount of shared alpha activity between the central region $(\mathrm{CZ})$ and the left frontal (F3) and right frontal (F1) areas. This metric helps to explain why frontal EEG alpha power asymmetry is significantly related to depression when analyzed with a vertex reference $(\mathrm{CZ})$ but not with a linked ears reference (A1/A2). The problem with using a vertex reference is that it is an active reference. If alpha power over the right frontal lobe is similar to that at the vertex, little net alpha activity will be recorded. Conversely, if left frontal alpha power is very different from that at $\mathrm{CZ}$, then a large differential amount of alpha power will be recorded. Frontal alpha power asymmetry using the $\mathrm{CZ}$ reference, is therefore, a measure of the relationship between frontal and central brain alpha on the left and right. 
Coherence, as defined here, is a measure of the similarity of frequency activity between two brain sites with a constant phase relationship. There was a high correlation between depression severity and alpha coherence asymmetry $(\mathrm{r}=.56)$. The correlation with $\mathrm{CZ}$ alpha power asymmetry was lower $(\mathrm{r}=.34)$. It may well be that coherence training to normalize frontal alpha coherence asymmetry will prove to be more effective than alpha power asymmetry training for remediation of depression. However, no studies have yet been reported. Though alpha coherence asymmetry and alpha power asymmetry have a fairly high correlation $(\mathrm{r}=.70)$, they are measuring somewhat different.parameters. Alpha coherence asymmetry may be a better metric for biofeedback training.

The second problem addressed by Lawson (2001) was the role of comorbid anxiety on QEEG metrics. Anxiety tends to be associated with reduced alpha power in the right frontal lobe in the majority of cases (Sackheim, Greenberg, Weiman, \& Gur (1982) and Gurnee (2000). Subjects who are both anxious and depressed may have coherence or power abnormalities with left or right preponderance. One, therefore, cannot reliably predict the direction of change produced by asymmetry training in this common type of patient. It is likely that additional specific training to decrease anxiety will be necessary to optimally help this group of patients. 
Leuchter, Uijtdehaage, O’Hara \& Mandelkern (1999) have described a_refined QEEG measure (cordance), which integrates information from absolute and relative power measures. This measure is highly correlated with $\mathrm{rCBF}$. This measure may be used in the future for predicting outcomes of treatment for depression.

IV. Treatment of Depression with Neurotherapy

There are no controlled studies of neurofeedback therapy for depression, and there are no published references in which QEEG was used to guide neurofeedback training of depression. The first neurofeedback approach was that of the Othmers (1994), based on the studies showing underactivation of the left hemisphere in depression. Decreasing theta $(4-7 \mathrm{~Hz})$ and simultaneously increasing beta2 (15-18 $\mathrm{Hz}$ ) at C3 was found to reduce depression in most patients (Othmer, 1994). For overaroused patients (anxiety, mania), right hemisphere training was done by decreasing 4-7 Hz and increasing 12-15 Hz activity at C4. Later, inhibition of 8-11 $\mathrm{Hz}$ activity was used by the Othmers(1997) to deal with underactivation states. In unstable states (such as manic-depressive or bipolar disorder), bipolar training has often been effective, (increasing beta2 $(15-18 \mathrm{~Hz})$ at T3/FPI and increasing SMR $(12-15 \mathrm{~Hz})$ at T4/FP1. They note that excessive beta training may cause agitation, anxiety, mania, obsessive thoughts, compulsive behaviors, anger, aggressiveness, pressure in the chest, or a sensation of crawling skin. Excessive SMR training may 
cause depression, irritability or loss of emotional control. The Othmers report that left-sided beta 2 training increases ego strength and sense of well-being, whereas right-sided SMR training increases awareness of internal body state and socialemotional awareness of others (Othmer, 1997). Our results have been similar when using their approach.

Baehr and Baehr (1997) have used alpha asymmetry reduction to treat depression. In one study, 3 of 6 patients improved on the Chambers and Beck Depression Inventory depression measures both immediately after training and at 1 and 5 years after neurofeedback training. This type of training was not effective in 2 of their bipolar patients. Hammond (2001) had similar results in an independent study of frontal asymmetry training using the ROSHI procedure. Eight medicationresistant patients were trained for an average of 10.4 hours, with no other therapy. Mean improvement in MMPI depression scale scores of $28.75 \mathrm{~T}$ scores was found. All but one patient improved, and at 1-year follow-up, improvement was maintained in all of those who improved. Putnam (2001) has reported successful treatment with neurofeedback of depression associated with a stroke. Schneider, Heimann, \& Mattes (2003)_have reported successful treatment of depression by down-regulating slow cortical potentials.

We report here our results using QEEG to guide neurofeedback in treating depressed patients, with two case histories to illustrate its utility. Our approach has 
been to identify the most significant abnormalities on QEEG and treat them with a series of protocols intended to normalize the specific abnormalities. If an abnormality is increased compared to normal, we downtrain it. If an abnormality is decreased compared to normal, we uptrain it. Usually 5-10 sessions are done for each abnormality.

Recently, we have been using FP02 training to decrease theta $(2-7 \mathrm{~Hz})$ and increase beta $(15-18 \mathrm{~Hz})$ with depressed patients. They usually report feeling less depressed. This site is just medial to the right eyebrow. Although not proven, FP02 beta training appears to be a promising addition to depression protocols. Fisher (2003a) (2003b) used this site, inhibiting delta and theta and rewarding alpha, to decrease fear, but she has not tried rewarding beta to decrease depression (personal communication).

V. Case Histories

\section{CASE 1 (UNIPOLAR DISORDER)}

This 41 year-old woman had a long history of chronic depression. Treatment with a variety of antidepressant drugs did not help her depression. Training was begun with a classic Othmer protocol: C3 beta (reward decrease of 2$7 \mathrm{~Hz}$ and increase of $15 \mathrm{~Hz}$ at $\mathrm{C} 3$-monopolar training). She noted some improvement in her depression by the second session. By the 10th session, her 
depression was gone. A total of 16 sessions was done. She has been depression free for over two years.

This type of blind (non QEEG guided) training ("C3 beta") has been effective in our clinic about $80 \%$ of the time in remediating long-standing, drugresistant depression.

\section{CASE II (BIPOLAR DISORDER) (QEEG-GUIDED)}

The client was a 61-year-old man with life-long severe mood swings and intermittent bouts of severe depression. Lithium therapy was ineffective. Several antidepressants were tried without lasting benefit. Treatment with Topamax helped the depression, but triggered a sustained manic episode, during which the client got into trouble at work and was discharged. He again became severely depressed and took an overdose of medications in an attempt to commit suicide. The initial QEEG showed an increase in relative power of 7-9 Hz activity over the frontal and central regions bilaterally and an increase in $8-9 \mathrm{~Hz}$ activity in both occipital regions. There was an increase in the relative power of 22-30 Hz activity in the left frontal/temporal regions. Decreased coherence was noted in alpha at $\mathrm{O} 1 / \mathrm{F} 7$, in beta at F4/F8, in theta at F7/F8, and in delta at F7/F8.

He first completed 10 sessions to decrease $19-30 \mathrm{~Hz}$ at FP1 and noted marked improvement with less anxiety, irritability, anger and depression. Four 
sessions were done to decrease 7-9 Hz and increase 15-18 Hz. Five sessions were done to decrease $2-7 \mathrm{~Hz}$ and to increase $15-18 \mathrm{~Hz}$ at 02 .

Upon completion of training, the client was no longer having either severe depression or manic episodes. He still had some anger related to his employment. He is in counseling to resolve these issues. He has stopped treatment with Cymbalta (anti-depressant) but continues on Depakote (mood stabilizer). The follow-up QEEG revealed resolution of the slow abnormalities. The excessive high frequency beta activity had resolved in the left frontal and temporal areas, though there was still some excess $27-30 \mathrm{~Hz}$ activity in the parietal, central and mid-frontal areas. The decreases in coherence on the first study had normalized, with the development of increased coherence in several connections in left hemisphere delta and right hemisphere beta. These new hypercoherent abnormalities may represent a strategy used by the brain to ameliorate the original abnormalities. They were not accompanied by the development of any new symptoms.

VI. Conclusions

Recent advances in the neurobiology of depression and anxiety have led to a better understanding of the roles of different brain areas in triggering depression, happiness, anxiety, or calmness. Activation (increasing 15-18 Hz) of the left frontal lobe generally triggers mood elevation, while hypoactivation (excess theta 
or alpha) of the left frontal lobe appears to cause failure of suppression of the subcortical structures that mediate the depressed state. Activation of the right orbital frontal lobe at $15-18 \mathrm{~Hz}$ can produce mood elevation. High frequency (21$30 \mathrm{~Hz}$ ) activity in the Higher frequency activation of the right orbital frontal area, the posterior cingulate, or the inferior parietal lobules can trigger anxiety or mania, while calmness is usually associated with deactivation of these areas.

Neurofeedback can be used to capitalize on these findings to remediate depression and anxiety and promote happiness and calmness. QEEG and LORETA can be used to characterize the particular areas that need to be addressed in an individual patient, thereby producing the desired effects more quickly and persistently.

Recently, we have been using FP02 training to decrease theta $(2-7 \mathrm{~Hz})$ and increase beta $(15-18 \mathrm{~Hz})$ with depressed patients and they report feeling less depressed. Although not proven, FP02 beta training appears to be a promising addition to depression protocols. Fisher (2003a, 2003b) used this site, inhibiting delta and theta and rewarding alpha, to decrease fear, but has not tried rewarding beta to decrease depression (personal communication).

\section{References}

Adolphs, R. J., \& Tranel, D. (2004). Emotion. In Principles and practice of behavioral neurology and neuropsychology (pp.451-474). Philadelphia: Saunders. 
Baehr, E., \& Baehr, R. (1997). The use of neurofeedback as adjunctive therapeutic treatment for depression: Three case studies. Biofeedback, 25, 10-11.

Baehr, E., Rosenfeld, P., Baehr, R., \& Earnest, C. (1999). Clinical use of an alpha asymmetry protocol in the treatment of depressive disorders. (pp.181-188).

Evans, J., \& Arbanel, A. (editors) in Introduction to QEEG and neurofeedback_New York: Academic Press.

Baehr, E., Rosenfeld, J. P., \& Baehr, R. (1997). The clinical use of an alpha asymmetry protocol in the neurofeedback treatment of depression: Two case studies. J Neurotherapy, 2, 10-23.

Davidson, R. J., \& Irwin, W. (1999). The functional neuroanatomy of emotion and affective style. Trends Cogn. Sci. 3, 11-22.

Earnest. C. (1999). Single case study of EEG asymmetry biofeedback for depression: an independent replication in an adolescent. J. Neurotherapy, 3, $38-35$.

Fisher, Sebern F. (2003). The fear protocol: The theory of FP02 and the implications of new clinical data. Winter Brain Abstracts.

Fisher, Sebern. F. (2003). Fear and FP02: The implication of a new protocol. ISNR proceedings. $11^{\text {th }}$ Annual Conference.

Gurnee, R. (2000). QEEG subtypes of anxiety. Abstract. Int. Soc. Neuronal Regulation. 
Hammond, D. C. (2001). Neurofeedback treatment of depression with the ROSHI. J. Neurotherap 4, 45-56.

Lawson. R. (2001). Anteriar alpha asymmetry in anxiety and depression. Abstract. Society for Neuronal Regulation.

Lawson, R., Rogers, R. Barnes, T., Bodenhamer-David, E., \& Reed, S. (2000).A comparison of exterior alpha asymmetry measures as predictors of depression severity. Abstract. Society for Neuronal Regulation.

Leuchter, A. F., Uijtdehaage, S. A. J., Cook, I. A., O’Hara, R., \& Mandelkern, N. (1999). Relationship between_brain electrical activity and cortical perfusion in normal subjects. Psychiat Res., 90, 125-140.

Lieber, A. L. \& Newbury, N. D. (1988). Diagnosis and subtyping of depressive disorders by QEEG discriminating IV. Subtypes of unipolar depression. Hillside J. Clin. Psychiat., 10, 73-82.

Liotti, M., \& Mayberg, H. S. (2001). The role of functional neuroimaging in the neoropsychology of depression. _J. Clin. Exp. Neuropsychol. 23, 121-136. Mayberg, H. S., Brannan, S. K., Tekell, J. L. Silva, J. A., Makurvin, R. K., \& McGinnis, S. (2000). Regional metabolic effects of fluoxetine in major depression: Serial changes and relationship to clinical depression. Psychiat. $48,830-843$. 
Ongur, D., Drevets. W. D. \& Price, J. L. (1998 ). Glial reduction in the subgenual prefrontal cortex in mood disorders. Proc. Nat. Acad. Sci. USA 95, 1329013295.

Othmer, S. (1994). Depression. In Training Syllabus. Vol. II, EEG Spectrum, Encino, CA.

Othmer, S. (1997). Depression. In Training Syllabus. Vol. II, EEG Spectrum, Encino, CA.

Prichep, L. S., Lieber, A. L., John, E. R. (1986). Quantitative EEG in depressive disorders. In Shagass, C. (ed.) Electrical brain potentials and psychotherapy, Amsterdam: Elesvier.

Putnam, J. A. (2001). EEG biofeedback in a female stroke patient with depression. J. Neurotherapy, 5, 27-38.

Rosenfeld P. J. (1999). An EEG biofeedback protocol for affective disorders. Clin. EEG,31, 7-12.

Rosenfeld, J. P., Baehr, E., Baehr, R. Gottlieb, I. H., \& Rangarndth, C. (1996). Preliminary evidence that daily changes in frontal alpha asymmetry correlate with changes in affect in therapy sessions. Int. J. Psychophysiol., 23, 137141. 
Sackheim, H. A., Greenberg., Weiman, A. C., Gur, R. C., \& Hungerbuhler, J. P. (1982). Hemispheric asymmetry in the expression of positive and negative emotions. Arch. Neurol. 39, 210-218.

Schneider, F., Heimann, H., Mattes, R. (2003). Self-regulation of slow cortical potentials in psychiatric patients. Biofeedback, 17, 2003-214.

Starkstein, S. E., \& Robinson, R. G. (1991). The roles of the frontal lobes in affective disorder following stroke. In Levison, A. S., Eisenberg, S. M., and Burton, A. (eds.) Frontal lobe function and dysfunction. (pp. 212-218): New York, Oxford University Press. 


\section{TABLE I}

DEPRESSIVE SUBTYPES AND ANXIETY

CLINICAL/QEEG CORRELATIONS AND NEUROFEEDBACK PROTOCALS

\begin{tabular}{|c|c|c|c|c|c|}
\hline & GENETIC & ACQUIRED & \begin{tabular}{|l|} 
RESPONSE TO \\
PSYCHOTHERAPY
\end{tabular} & $\begin{array}{l}\text { ASSOCIATED } \\
\text { WITH QEEG } \\
\text { ABNORMALITY }\end{array}$ & \begin{tabular}{|l} 
Symptom- \\
Based \\
NF \\
TRAINING \\
PROTOCOLS
\end{tabular} \\
\hline $\begin{array}{l}1 . \\
\text { Endogenous } \\
\text { depression } \\
\text { (unipolar) }\end{array}$ & $\sqrt{ }$ & & POOR & $\begin{array}{l}\text { Slowing F8 (trait } \\
\text { marker) }\end{array}$ & $\begin{array}{c}\downarrow 2-7 / \uparrow 15- \\
18 \text { F8 }\end{array}$ \\
\hline $\begin{array}{l}\text { 2. Cognitive } \\
\text { depression } \\
\text { (unipolar) }\end{array}$ & $\sqrt{ }$ & & YES & Slowing F7 (trait) & $\begin{array}{c}\downarrow 2-7 / \uparrow 15- \\
18 \text { F7 }\end{array}$ \\
\hline $\begin{array}{l}\text { 3. Manic/ } \\
\text { depressive } \\
\text { (bipolar) }\end{array}$ & $\sqrt{ }$ & & POOR & $\begin{array}{l}\text { Slowing F3 (trait } \\
\text { marker for } \\
\text { depression) } \\
\text { Excess frontal } \\
\text { beta (trait marker } \\
\text { for mania) }\end{array}$ & $\begin{array}{c}\text { Combined } \\
\text { training } \\
\downarrow \\
\text { stabilized }\end{array}$ \\
\hline $\begin{array}{l}\text { 4. Reactive } \\
\text { depression }\end{array}$ & & $\sqrt{ }$ & YES & $\begin{array}{l}\text { No trait markers } \\
\text { Alpha asymmetry, } \\
\text { L> R (CZ } \\
\text { reference) } \\
\text { (state marker) }\end{array}$ & $\begin{array}{l}\text { Normalize } \\
\text { alpha } \\
\text { power or } \\
\text { coherence } \\
\text { asymmetry }\end{array}$ \\
\hline 5. Anxiety & $\sqrt{ }$ & $\sqrt{ }$ & POOR & $\begin{array}{l}\text { Excess of high } \\
\text { beta }(20-30 \mathrm{~Hz}) \\
\text { Any brain area } \\
\text { (may be trait or } \\
\text { state marker) }\end{array}$ & \begin{tabular}{|l|} 
Decrease \\
high- \\
frequency \\
beta \\
(whatever \\
location) \\
\end{tabular} \\
\hline & & & & & \\
\hline
\end{tabular}




\section{TABLE II}

FRONTAL RHYTHM

Delta ( $R$ or L)

Theta (R or L)

Alpha (R or L)

Beta 1 (12-15 Hz) (R)

Beta 2 (15-18 Hz) (L)

Beta 3 (19-30 Hz) (R or L)

\section{BEHAVIORAL STATE}

Sleepy

Drowsiness

Awake, Calm, Unfocused

Calm, observant

Fully focused, attentive, less depressed

Anxious, irritable 
